\section{Efeitos da hidroginástica sobre a capacidade funcional de idosos: metanálise de estudos randomizados}

\section{Effects of water based exercise on the functional capacity in older adults: meta- analysis of randomized trials}

\author{
Thaís Reichert ${ }^{1}$ \\ Alexandre Konig Garcia Prado \\ Ana Carolina Kanitz ${ }^{2}$ \\ Luiz Fernando Martins Kruel ${ }^{1}$
}

\section{RESUMO}

Os efeitos deletérios do envelhecimento limitam a capacidade do idoso de realizar atividades básicas. Nesse sentido, é relevante saber se a hidroginástica, modalidade fortemente recomendada ao público idoso, promove independência funcional a essa população. $\mathrm{O}$ objetivo dessa revisão sistemática e metanálise foi avaliar os efeitos da prática de hidroginástica sobre a capacidade funcional de idosos. A busca foi realizada na PUBMED, Scielo, Cochrane, PEDro e de forma manual em agosto de 2015. Dois revisores independentes selecionaram os estudos com base em critérios previamente definidos (ensaios clínicos randomizados que avaliaram o efeito da prática de hidroginástica sobre o desempenho em testes funcionais em idosos acima de 60 anos saudáveis). A metanálise foi realizada usando modelo de efeitos aleatórios e a heterogeneidade estatística foi avaliada pelo teste Q de Cochran e inconsistência $\left(\mathrm{I}^{2}\right)$. De um total de 7056 artigos, oito estudos clínicos randomizados foram incluídos com 301 sujeitos. A hidroginástica promove um aumento significativo na força muscular resistente de membros superiores (7,63 [intervalo de confiança de $95 \%$, 4,19 a 11,08]) e inferiores (6,65 [intervalo de confiança de $95 \%, 3,23$ a 10,07]), na flexibilidade de membros superiores (3,75 [intervalo de confiança de 95\%, 0,39 a 7,11]) e inferiores $(6,10$ [intervalo de confiança de $95 \%, 0,32$ a 11,89 ] $)$ e no equilíbrio dinâmico $(0,95$ [intervalo de confiança de $95 \%, 1,45$ a 0,44$]$ ) de idosos. Conclui-se que a hidroginástica pode ser indicada como uma forma de melhorar a capacidade funcional e independência de idosos.

\section{PALAVRAS-CHAVE}

Envelhecimento; Exercício; Força muscular .

\begin{abstract}
The deleterious effects of aging limit the elderly's ability to perform basic activities. Therefore, it is important to know if water based exercise, strongly recommended to olders, promotes functional independence to this population. The aim of this systematic review and meta-analysis was to evaluate the effects of water based exercise on the functional capacity in older adults. The search was performed in PubMed, SciELO, Cochrane, PEDro and manually in August 2015. Two independent reviewers assessed the eligibility of studies based on predefined inclusion criteria (randomized clinical trials that evaluated the effects of water based exercise on performance in functional tests in over 60 bealthy elderly). The meta-analysis was performed using random-effects model. Statistical heterogeneity was assessed by Cochran $Q$ test and inconsistency $\left(I^{2}\right)$. Of the total of 7056 titles, eight randomized clinical trials were included with 301 subjects. Water based exercise promotes significant increase in endurance strength of upper (7.63 [95\% confidence interval, 4.19 to 11.08]) and lower limbs (6.65 [95\% confidence interval, 3.23 to 10.07]), in flexibility of upper (3.75 [95\% confidence interval, 0.39 to 7.11]) and lower limbs (6.10 [95\% confidence interval, 0.32 a 11.89]) and dynamic balance (0.95 [95\% confidence interval, 1.45 a 0.44]). We concluded that water based exercise can be indicated to improve the functional capacity and independence of older adults.
\end{abstract}

\section{KEYWORDS}

Aging; Exercise; Muscle Strength
Rev Bras Ativ Fís Saúde p. 447-457 DOI

http://dx.doi.org/10.12820/rbafs.v.20n5p447

1 Universidade Federal do Rio Grande do Sul, Porto Alegre, Rio Grande do Sul, Brasil

2 Universidade Federal de Uberlândia, Uberlândia, Minas Gerais, Brasil 


\section{INTRODUCCÃO}

A população idosa tem apresentado um crescimento acelerado nas últimas décadas ${ }^{1}$. Projeções da Organização Mundial das Nações Unidas (ONU) indicam que essa população continuará em progressivo crescimento, de modo que, em 2050, o número de idosos chegará a $21 \%$ da população mundial total, superando inclusive o número de jovens. Neste mesmo ano, estima-se que a expectativa de vida do brasileiro possa alcançar 81,2 anos ${ }^{2}$.

$\mathrm{O}$ avanço da idade gera importantes mudanças fisiológicas e biomecânicas, como a diminuição da capacidade aeróbia ${ }^{3}$, da força muscular ${ }^{4}$, do equilíbrio ${ }^{5}$ e da flexibilidade, limitando a amplitude de movimento ${ }^{6}$. Esses efeitos deletérios do processo de envelhecimento limitam a funcionalidade do idoso, repercutindo sobre a sua capacidade de realizar as atividades de vida diária (AVDs) ${ }^{7}$. Sendo assim, a limitação funcional torna essa população cada vez mais vulnerável e frequentemente dependente de outras pessoas para realizar atividades básicas como caminhar, subir degraus e levantar da cadeira, o que, por sua vez, reduz o nível de atividade física desses sujeitos.

Desta forma, diante de um progressivo crescimento da população idosa e aumento da expectativa de vida, fatores estes que tendem a resultar em um maior número de idosos dependentes, torna-se de fundamental importância identificar intervenções que possibilitem a prevenção da incapacidade e a otimização da independência desse público ${ }^{4}$. Nesse sentido, estudos têm demonstrado o efeito benéfico do exercício físico como uma contramedida eficiente para melhorar a capacidade funcional do indivíduo, contribuindo para uma maior capacidade de realizar as AVDs. Dentro deste contexto, exercícios físicos realizados em meio aquático, como a hidroginástica, têm sido especialmente recomendados para a população idosa por apresentarem um reduzido impacto articular em membros inferiores ${ }^{8}$, preservando as articulações, e menores valores de pressão arterial e frequência cardíaca durante a imersão ${ }^{9}$, possibilitando uma maior segurança para o praticamente.

Estudos da literatura já avaliaram os efeitos da prática de hidroginástica sobre a capacidade funcional de idosos, porém os seus resultados são controversos $^{4,10-16}$. Uma revisão sistemática demonstrou uma forte evidência de que a hidroginástica promove ganhos de força e na capacidade cardiorrespiratória e evidência moderada de ganhos na flexibilidade ${ }^{17}$. Contudo, a conclusão deste estudo foi baseada fundamentalmente em avaliações diretas cujos resultados não refletem diretamente a capacidade do indivíduo de realizar as AVDs. Além disso, a busca na literatura deste estudo foi restringida a estudos publicados entre 1985 a 2009 e não foi realizada uma metanálise.

Sendo assim, ainda não há uma evidência robusta e quantitativa sobre os efeitos da prática de hidroginástica na capacidade funcional de idosos. Esta é uma questão pertinente, visto que o principal público de aulas de hidroginástica em academias é a população idosa e, ainda, esta modalidade é fortemente recomendada por profissionais da saúde para essa faixa etária.

Desta forma, o objetivo do presente estudo foi conduzir uma revisão sistemática e metanálise de ensaios clínicos randomizados para avaliar os efeitos da prática de hidroginástica sobre a capacidade funcional de indivíduos idosos. 


\section{MÉTODOS}

Esta revisão sistemática seguiu as recomendações propostas pela Colaboração Cochrane $^{18}$ e pelo Preferred Reporting Items for Systematic Review and Meta-analyses: The PRISMA Statement ${ }^{19}$.

\section{Critérios de elegibilidade}

Foram incluídos somente ensaios clínicos randomizados que avaliaram o efeito da prática de hidroginástica sobre a capacidade funcional de idosos acima de 60 anos saudáveis de ambos os sexos, sem limite superior de idade. Consideraram-se os estudos em que a intervenção possuía caráter aeróbio, de força ou combinado. Além disso, os estudos deveriam apresentar comparações dos resultados da intervenção em meio aquático com indivíduos que: não realizaram nenhum tipo de exercício (Grupo Controle) ou realizaram intervenção fora do ambiente aquático ou também realizaram intervenção no meio aquático. Foram incluídos somente estudos que avaliaram o desempenho dos idosos nos testes funcionais levantar e sentar, flexão de cotovelo, sentado e alcançar, alcançar atrás das costas, caminhada de seis minutos, foot up and go e timed up and go. Por fim, apenas os estudos cujos desfechos não apresentassem diferença significativa entre os grupos no momento pré-treinamento foram incluídos.

\section{Estratégias de busca}

As seguintes bases de dados foram consultadas sem limite de datas: PUBMED, Scielo, Cochrane e PEDro em agosto de 2015. Adicionalmente foi realizada uma busca manual na lista de referências dos estudos encontrados nas bases de dados. A busca foi restringida a estudos publicados em português, espanhol ou inglês. Os seguintes termos foram utilizados de forma combinada e/ou isolada: "Water exercise", "Aquatic Exercise", "Water-based exercise", "Aged", "Elderly", "Aging”, "Older".

\section{Seleção de estudos e extração de dados}

Dois pesquisadores independentemente avaliaram os títulos e resumos de todos os artigos encontrados pela estratégia de busca. Os estudos cujos resumos não forneceram informações suficientes para identificação dos critérios de inclusão foram lidos na íntegra. Os mesmos dois pesquisadores independentemente realizaram a extração dos dados considerando as características metodológicas dos estudos, intervenções e desfechos observados. Discordâncias entre os dois pesquisadores foram solucionadas por consenso e, caso fosse necessário, por um terceiro pesquisador.

\section{Avaliação do risco de viés}

A avaliação da qualidade dos estudos incluiu adequado método de randomização, alocação sigilosa, cegamento dos avaliadores, análise com intenção de tratar, descrição de perdas e exclusões, apresentação adequada dos resultados e cálculo do tamanho amostral. Dois revisores independentes realizaram a avaliação do risco de viés e para cada critério, os estudos foram classificados em alto risco, baixo risco ou risco não claro.

\section{Análise estatística}


Todas as análises foram conduzidas usando o software Review Manager 5.3 (Colaboração Cochrane $)^{18}$. A metanálise foi realizada usando modelo de efeitos aleatórios e as medidas de efeito foram obtidas pelos valores da média, desvio padrão e $\mathrm{n}$ amostral do pós-intervenção. A heterogeneidade estatística do efeito do tratamento entre os estudos foi avaliada por meio do teste $\mathrm{Q}$ de Cochran e do teste de inconsistência $\left(\mathrm{I}^{2}\right)$. Foi adotado um $\alpha=0,05$.

\section{RESULTADOS}

\section{Descrição dos estudos}

A partir da busca inicial, 7065 artigos foram encontrados, sendo que a leitura dos títulos e resumos resultou na exclusão de 7045, restando 20 artigos para a leitura completa. Destes, oito preencheram os critérios de elegibilidade e foram incluídos na presente revisão (Figura 1).

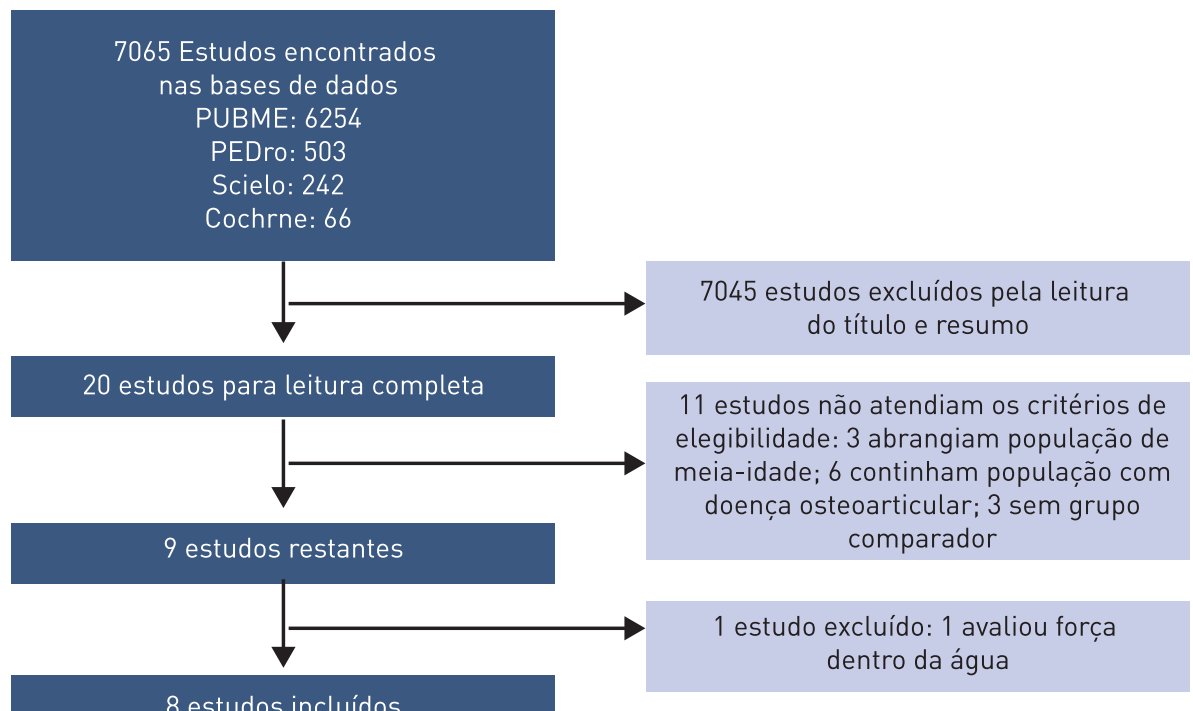

8 estudos incluídos

FIGURA 1 - Fluxogram da busca e an[alise de artigos nas diferentes fases de revisão.

Um sumário das características dos estudos incluídos pode ser visualizado no quadro 1. Os estudos incluídos acumularam um "n" total de 301 participantes, 187 alocados no grupo intervenção hidroginástica e 114 no grupo comparador.

\section{Análise do risco de viés}

A avaliação do risco de viés de cada estudo pode ser visualizada no quadro 2. Em todos os estudos, a alocação dos sujeitos entre os grupos foi realizada por meio da randomização, contudo nenhum deles descreve de que forma este processo foi realizado. Além disso, em nenhum estudo o processo de randomização foi realizado por alguém sem envolvimento direto na pesquisa e as avaliações foram realizadas por avaliadores cegados. Em relação à análise por intenção de tratar, um estudo (12,5\%) não teve perda amostral, avaliando a totalidade da amostra no pré e pós-período de treinamento. Já os que apresentaram perda amostral, a maioria reporta o motivo (5 estudos, 62,5\%). Todos os estudos apresentaram os resultados adequadamente (8 estudos). Por fim, apenas um estudo (12,5\%) realizou o cálculo do $\mathrm{n}$ amostral antes da realização da pesquisa. 
QUADRO 1 - Características dos estudos incluídos

\begin{tabular}{|c|c|c|c|c|c|}
\hline \multirow{2}{*}{ Estudo } & \multirow{2}{*}{ Amostra } & \multirow{2}{*}{ Intervenção } & \multirow{2}{*}{ Desfecho } & \multicolumn{2}{|c|}{ Intervenção } \\
\hline & & & & Pré & Pós \\
\hline \multirow{6}{*}{$\begin{array}{l}\text { Alves et } \\
\text { al. }^{10}\end{array}$} & \multirow{6}{*}{$\begin{array}{l}\text { Idosas sedentárias } \\
\text { Grupo experimental: } \\
30 \text { indivíduos ( } 78 \pm 30 \text { anos) } \\
\text { Grupo controle: } \\
30 \text { indivíduos ( } 79 \pm 50 \text { anos) }\end{array}$} & \multirow{6}{*}{$\begin{array}{l}\text { Duração: } 12 \text { semanas } \\
\text { Frequência: } 2 x / \text { semana } \\
\text { Duração da sessão: } 45 \text { min } \\
\text { Protocolo: aeróbio + força/ } \\
\text { resistência } \\
\text { Intensidade: não informada }\end{array}$} & Levantar e sentar (rep) & $8,71 \pm 1,6$ & $14,9 \pm 1,8^{*}$ \\
\hline & & & Flexão de antebraço (rep) & $12,2 \pm 2,5$ & $21,6 \pm 2,8^{*}$ \\
\hline & & & Sentado e alcançar $(\mathrm{cm})$ & $-5,6 \pm 7,5$ & $5,2 \pm 9,2^{*}$ \\
\hline & & & Foot up and go (s) & $7,3 \pm 1,5$ & $5,8 \pm 1,0^{*}$ \\
\hline & & & Alcançar atrás das costas $(\mathrm{cm})$ & $-11,1 \pm 10,9$ & $-1,1 \pm 7,6^{*}$ \\
\hline & & & Caminhada 6 minutos (m) & $419,8 \pm 72,4$ & $513,0 \pm 83,6^{*}$ \\
\hline \multirow{4}{*}{$\begin{array}{l}\text { Bento et } \\
\text { al. }^{11}\end{array}$} & \multirow{4}{*}{$\begin{array}{l}\text { Homens e mulheres idosos e } \\
\text { inativos. } \\
\text { Grupo experimental: } \\
3 \text { homens e } 21 \text { mulheres }(65,6 \pm 4,2 \\
\text { anos). } \\
\text { Grupo controle: } \\
4 \text { homens e } 10 \text { mulheres }(65,6 \pm 4,4)\end{array}$} & \multirow{4}{*}{$\begin{array}{l}\text { Duração: } 12 \text { semanas } \\
\text { Frequência: } 3 x / \text { semana } \\
\text { Duração da sessão: } 60 \text { min } \\
\text { Protocolo: aeróbio + força } \\
\text { Intensidade: } 12 \text { a } 16 \text { de Borg } \\
\text { (6-20) }\end{array}$} & Levantar e sentar (rep) & $13,8 \pm 2,8$ & $14,6 \pm 2,1$ \\
\hline & & & Sentado e alcançar (cm) & $1,7 \pm 10,8$ & $5,3 \pm 11,9 *$ \\
\hline & & & Foot up and go (s) & $5,5 \pm 0,8$ & $5,1 \pm 0,5^{*}$ \\
\hline & & & Caminhada de 6 minutos (m) & $572,7 \pm 75,1$ & $596,8 \pm 77,1^{*}$ \\
\hline \multirow{3}{*}{$\begin{array}{l}\text { Bergamin } \\
\text { et al. }{ }^{12}\end{array}$} & \multirow{3}{*}{$\begin{array}{l}\text { Homens ( } 72,1 \pm 6,1 \text { anos) e mulheres } \\
\text { ( } 72,3 \pm 4,7 \text { anos) acima de } 65 \text { anos } \\
\text { sedentários. } \\
\text { Grupo experimental: } 17 \text { indivíduos. } \\
\text { Grupo controle: } 19 \text { indivíduos }\end{array}$} & \multirow{3}{*}{$\begin{array}{l}\text { Duração: } 24 \text { semanas } \\
\text { Frequência: } 2 x / \text { semana } \\
\text { Duração da sessão: } 60 \text { min } \\
\text { Protocolo: aeróbio Intensidade: } \\
13 \text { a } 16 \text { de Borg }(6-20)\end{array}$} & Alcançar atrás das costas $(\mathrm{cm})$ & $-7,8 \pm 8,9$ & $-5,8 \pm 8,3^{*}$ \\
\hline & & & Sentado e alcançar (cm) & $3,2 \pm 7,3$ & $13,0 \pm 6,8^{*}$ \\
\hline & & & Foot up and go (s) & $5,6 \pm 1,2$ & $4,5 \pm 0,8^{*}$ \\
\hline \multirow{5}{*}{$\begin{array}{l}\text { Bocalini et } \\
\text { al.13 }\end{array}$} & \multirow{5}{*}{$\begin{array}{l}\text { Mulheres idosas entre } 62 \text { e } 75 \text { anos } \\
\text { sedentárias } \\
\text { Grupo experimental: } 25 \text { indivíduos } \\
\text { Grupo controle: } 10 \text { indivíduos }\end{array}$} & \multirow{5}{*}{$\begin{array}{l}\text { Duração: } 12 \text { semanas } \\
\text { Frequência: } 3 x / \text { semana } \\
\text { Duração da sessão: } 60 \text { min } \\
\text { Protocolo: aeróbio } \\
\text { Intensidade: } 70 \% \text { da } \mathrm{FC}_{\max } \\
\text { predita pela idade }\end{array}$} & Levantar e sentar (rep) & $24,0 \pm 3,0$ & $37,0 \pm 3,0 *$ \\
\hline & & & Flexão de cotovelo (rep) & $17,0 \pm 3,0$ & $25,0 \pm 1,0 *$ \\
\hline & & & Foot up and go (s) & $8,4 \pm 2,3$ & $5,5 \pm 1,0 *$ \\
\hline & & & Sentado e alcançar $(\mathrm{cm})$ & $24,0 \pm 3,0$ & $36,0 \pm 2,0^{*}$ \\
\hline & & & Alcançar atrás das costas $(\mathrm{cm})$ & $-10,0 \pm 2,0$ & $-6,0 \pm 2,0 *$ \\
\hline \multirow{4}{*}{$\begin{array}{l}\text { Katsura et } \\
\text { al. }^{14}\end{array}$} & \multirow{4}{*}{$\begin{array}{l}\text { Homens e mulheres sedentários } \\
\text { acima de } 65 \text { anos } \\
\text { Grupo experimental com } \\
\text { equipamento: } \\
1 \text { homem e } 11 \text { mulheres }(68,5 \pm 4,2 \\
\text { anos). } \\
\text { Grupo experimental sem } \\
\text { equipamento: } 3 \text { homens e } 5 \\
\text { mulheres }(70,0 \pm 5,1 \text { anos) }\end{array}$} & \multirow{4}{*}{$\begin{array}{l}\text { Duração: } 8 \text { semanas } \\
\text { Frequência: } 3 x / \text { semana } \\
\text { Duração da sessão: } 90 \text { min } \\
\text { Protocolo: aeróbio + força } \\
\text { Intensidade: moderadamente } \\
\text { forte (Borg). }\end{array}$} & Sentado e alcançar (cm) & $27,9 \pm 8,3$ & $31,3 \pm 6,7^{*}$ \\
\hline & & & TUG (s) & $5,9 \pm 0,7$ & $5,2 \pm 0,5^{*}$ \\
\hline & & & Caminhar 5 metros (s) & $3,8 \pm 0,6$ & $3,2 \pm 0,3^{*}$ \\
\hline & & & 10 metros com obstáculos (s) & $8,6 \pm 1,5$ & $8,3 \pm 0,9$ \\
\hline \multirow{3}{*}{$\begin{array}{l}\text { Rica et } \\
\text { al. }^{15}\end{array}$} & \multirow{3}{*}{$\begin{array}{l}\text { Mulheres idosas sedentárias } \\
\text { Grupo experimental: } 24 \text { indivíduos } \\
\text { Grupo controle: } 6 \text { indivíduos }\end{array}$} & \multirow{3}{*}{$\begin{array}{l}\text { Duração: } 12 \text { semanas } \\
\text { Frequência: } 3 x / \text { semana } \\
\text { Duração da sessão: } 60 \text { min } \\
\text { Exercícios: aeróbio } \\
\text { Intensidade: } 70 \% \text { da } \mathrm{FC}_{\max } \\
\text { predita pela idade }\end{array}$} & Flexão de cotovelo (rep) & $21,0 \pm 2,0$ & $32,0 \pm 1,0 *$ \\
\hline & & & Levantar e sentar (rep) & $24,0 \pm 1,0$ & $35,0 \pm 1,0^{*}$ \\
\hline & & & $\begin{array}{l}\text { Caminhada/corrida } 800 \text { metros } \\
\text { (min) }\end{array}$ & $16,0 \pm 1,0$ & $10,0 \pm 1,0^{*}$ \\
\hline \multirow{4}{*}{$\begin{array}{l}\text { Sanders } \\
\text { et al. }{ }^{16}\end{array}$} & \multirow{4}{*}{$\begin{array}{l}\text { Mulheres idosas com menos de } 150 \\
\text { min/semana de atividade física } \\
\text { Grupo experimental: } 43 \text { indivíduos } \\
\text { (73,6 } \pm 13,5 \text { anos) } \\
\text { Grupo controle: } 17 \text { indivíduos } \\
\text { (72,8 } \pm 27,4 \text { anos) }\end{array}$} & \multirow{4}{*}{$\begin{array}{l}\text { Duração: } 16 \text { semanas } \\
\text { Frequência: } 3 x / \text { semana } \\
\text { Duração da sessão: } 20-45 \text { min } \\
\text { Protocolo: AVDs + aeróbio + } \\
\text { força } \\
\text { Intensidade: fácil - muito } \\
\text { intenso (Borg) }\end{array}$} & Sentado e alcançar (cm) & $25,6 \pm 6,5$ & $27,7 \pm 6,9 *$ \\
\hline & & & Levantar e sentar (rep) & $10,8 \pm 3,1$ & $14,1 \pm 3,9 *$ \\
\hline & & & Foot up and go (s) & $35,3 \pm 10,7$ & $28,1 \pm 9,6^{*}$ \\
\hline & & & Flexão de cotovelo (rep) & $14,0 \pm 3,8$ & $19,5 \pm 5,0 *$ \\
\hline \multirow{7}{*}{$\begin{array}{l}\text { Tsourlou } \\
\text { et al. }{ }^{4}\end{array}$} & eres idosas moderadamente & Duração: 24 semanas & Sentar e alcançar (cm) & $21,1 \pm 1,9$ & $23,6 \pm 1,8^{*}$ \\
\hline & ativas. & Frequência: 3x/semana & & & \\
\hline & Grupo experimental: 12 indivíduos & Duração da sessão: 60 min & & & \\
\hline & $(69,3 \pm 1,9$ anos $)$ & Protocolo: aeróbio + força & & & \\
\hline & $\begin{array}{l}\text { Grupo controle: } 10 \text { indivíduos } \\
(68,4 \pm 6,7 \text { anos })\end{array}$ & $\begin{array}{l}\text { Intensidade: Aeróbio: 65-80\% } \\
\text { da FCmax }\end{array}$ & TUG (s) & $6,3 \pm 0,3$ & $5,1 \pm 0,2^{*}$ \\
\hline & & Força: 12 a 15 repetições, 60- & & & \\
\hline & & 120 bpm & & & \\
\hline
\end{tabular}

min: minutos; rep: repetições; cm: centímetros; s: segundos; m: metros; TUG: timed up and go; FC frequência cardíaca máxima; AVDs: atividades de vida diária; bpm: batidas por minuto. * indica diferença significativa do pré para o pós-treinamento 


\begin{tabular}{|c|c|c|c|c|c|c|c|}
\hline Estudo & $\begin{array}{l}\text { Geração de } \\
\text { sequência } \\
\text { aleatória }\end{array}$ & $\begin{array}{l}\text { Sigilo de } \\
\text { alocação }\end{array}$ & $\begin{array}{l}\text { Cegamento } \\
\text { dos } \\
\text { avaliadores }\end{array}$ & $\begin{array}{c}\text { Análise por } \\
\text { intenção de } \\
\text { tratar }\end{array}$ & $\begin{array}{l}\text { Descrição } \\
\text { das perdas } \\
\text { amostrais }\end{array}$ & $\begin{array}{l}\text { Descrição dos } \\
\text { resultados }\end{array}$ & $\begin{array}{c}\text { Cálculo do } \\
\text { tamanho } \\
\text { amostral }\end{array}$ \\
\hline Alves et al. ${ }^{10}$ & + & + & + & + & - & - & - \\
\hline Bento et al. ${ }^{11}$ & $?$ & + & + & + & - & - & $?$ \\
\hline Bergamin et al..$^{12}$ & $?$ & + & + & + & - & - & + \\
\hline Bocalini et al. ${ }^{13}$ & + & + & + & + & + & - & + \\
\hline Katsura et al. ${ }^{14}$ & $?$ & + & + & - & - & - & + \\
\hline Rica et al. ${ }^{15}$ & + & + & + & + & + & - & + \\
\hline Sanders et al. ${ }^{16}$ & + & + & + & + & - & - & + \\
\hline Tsourlou et al. ${ }^{4}$ & + & + & + & + & - & - & + \\
\hline
\end{tabular}

+: alto risco; -: baixo risco; ?: risco não claro

\section{Efeitos da intervenção}

Sete estudos compararam os efeitos do treinamento de hidroginástica a um grupo controle (que continuaram suas atividades normalmente) e um comparou dois programas de treinamento de hidroginástica (com e sem o uso de equipamentos). Da totalidade de estudos avaliados, dois ${ }^{11,14}$ observaram que a prática de hidroginástica promoveu melhoras significativas em parte dos desfechos analisados, enquanto que seis ${ }^{10,12,13,15-17}$ encontraram melhoras significativas em todos os testes funcionais realizados.

Para a realização da metanálise, foram incluídos apenas os estudos que compararam o treinamento de hidroginástica a um grupo controle.

$\mathrm{Na}$ avaliação do desfecho flexão de cotovelo, quatro estudos foram incluídos ${ }^{10,13,15,16}$. A metanálise demonstrou que a prática de hidroginástica promove um incremento significativo desse parâmetro $(7,63$; IC 95\%: 4,19 a 11,08; I²: 96\%). Contudo, uma alta heterogeneidade entre os estudos foi encontrada, que pode ser atribuída aos estudos de Alves et al. ${ }^{10}$ e Rica et al. ${ }^{15}$, que não utilizaram equipamentos resistivos durante as aulas. A retirada destes estudos tornou a heterogeneidade nula, permanecendo a melhora significativa (Figura 2a).

Para a avaliação do teste levantar e sentar, cinco estudos foram incluí$\operatorname{dos}^{10,11,13,15,16}$. Demonstrou-se que ganhos significativos nesse desfecho são proporcionados pela prática de hidroginástica $(6,65$; IC 95\%: 3,23 a 10,07; I²: $97 \%$ ). Devido à alta heterogeneidade encontrada, realizou-se uma análise de sensibilidade incluindo apenas os estudos que descreveram um treinamento de força estruturado em seu protocolo de treinamento ${ }^{11,16}$. Essa análise demonstrou uma heterogeneidade nula e manutenção do efeito positivo e significativo da hidroginástica sobre o teste levantar e sentar (Figura 2b).

Apenas dois estudos foram incluídos na metanálise para análise do desfecho capacidade cardiorrespiratória ${ }^{10,11}$. Demonstrou-se que a hidroginástica não promove uma melhora significativa deste parâmetro. Contudo, este resultado deve ser analisado com cuidado, devido ao pequeno número de estudos incluídos na análise e a alta heterogeneidade existente entre eles (Figura 3).

Três estudos foram incluídos para a metanálise do teste alcançar atrás das $\operatorname{costas}^{10,12,13}$, em que demonstrou-se um aumento significativo após o treinamento de hidroginástica $(3,75$; IC $95 \%: 0,39$ a 7,11 ; I²: $61 \%)$. A heterogeneidade foi reduzida para $43 \%$, sem alterar o resultado da metanálise, após a exclusão do estudo de Bergamin et al. ${ }^{12}$ (Figura 4a). 


\begin{tabular}{|c|c|c|c|c|c|c|c|}
\hline \multirow{2}{*}{$\begin{array}{l}\text { a } \\
\text { Study or Subgroup }\end{array}$} & \multicolumn{3}{|c|}{ Experimental } & \multicolumn{3}{|c|}{ Control } & \multirow[b]{2}{*}{ Weight } \\
\hline & Mean & SD & Total & Mean & SD & Total & \\
\hline Alves et al. 2004 & 21.6 & 2.1 & 30 & 10.5 & 2.7 & 30 & $25.5 \%$ \\
\hline Bocalini et al. 2008 & 25 & 1 & 25 & 21 & 2 & 10 & $25.4 \%$ \\
\hline Rica et al. 2013 & 32 & 1 & 24 & 22 & 1 & 6 & $25.9 \%$ \\
\hline Sanders et al. 2013 & 19.5 & 5 & 43 & 14.3 & 4.1 & 17 & $23.2 \%$ \\
\hline Total $(95 \% \mathrm{Cl})$ & & & 122 & & & 63 & $100.0 \%$ \\
\hline
\end{tabular}

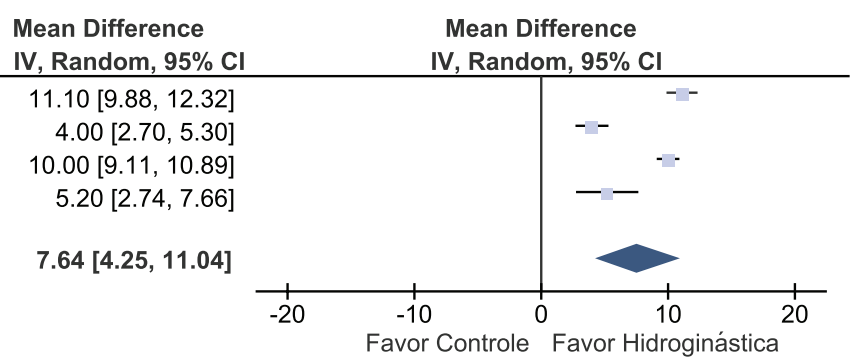

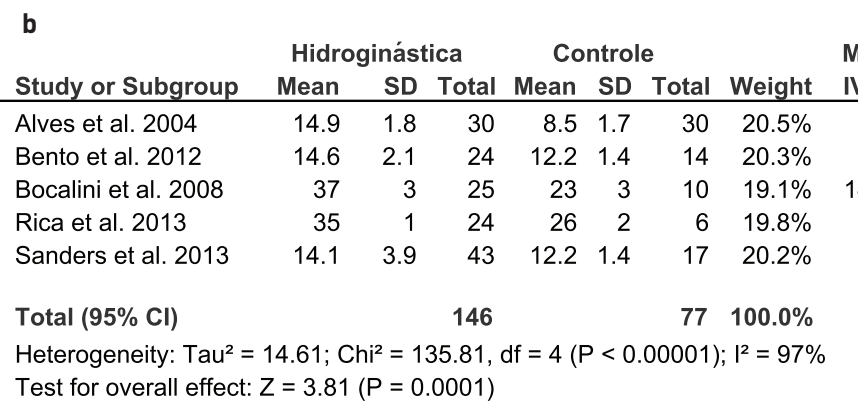

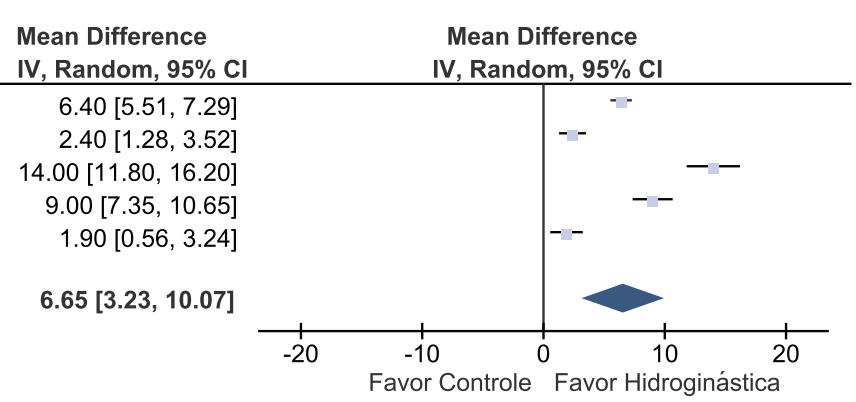

FIGURA 2 - Efeito da hidroginástica no teste funcional flexão de cotovelo (a) e sentar e levantar da cadeira (b).

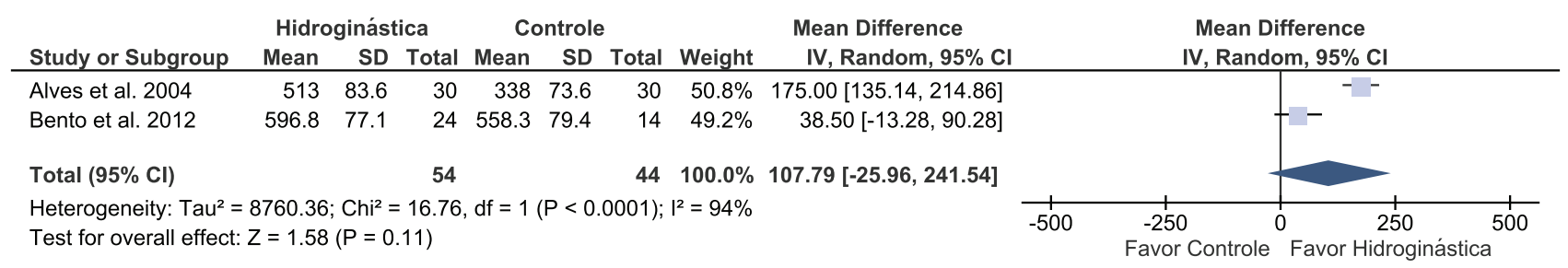

FIGURA 3 - Efeito da hidroginástica no teste funcional caminhada de seis minutos.

$\mathrm{Na}$ análise do teste sentado e alcançar, seis estudos foram incluídos ${ }^{10-13,16,17}$. A metanálise demonstrou que a hidroginástica gera ganhos significativos nessa variável (6,10; IC 95\%: 0,32 a 11,89; I²: 96\%). A heterogeneidade se tornou nula após retirada dos estudos de Alves et al. ${ }^{10}$, Bocalini et al. ${ }^{13}$ e Tsourlou et al. ${ }^{4}$, mantendo os efeitos positivos e significativos da hidroginástica sobre o teste sentado e alcançar (figura $4 b$ ).

Para a metanálise do teste foot up and go, sete estudos foram incluídos ${ }^{10-14,16,17}$. Os resultados apontam para um efeito positivo do treinamento de hidroginástica na redução do tempo necessário para realização do teste $(-0,95$; IC $95 \%$ : $-1,45$ a $\left.-0,44 ; I^{2}: 85 \%\right)$. A retirada dos estudos de Bento et al. ${ }^{11}$, Bocalini et al. ${ }^{13}$ e Katsura et al. ${ }^{14}$ tornou a heterogeneidade entre os estudos nula e manteve o efeito benéfico e significativo da hidroginástica sobre este parâmetro (Figura 5).

\section{DISCUSSÃO}

O presente estudo teve como objetivo revisar sistematicamente e realizar uma metanálise a fim de avaliar os efeitos da prática de hidroginástica sobre a capacidade funcional de idosos. Oito estudos foram encontrados, dos quais todos verificaram melhoras significativas após o treinamento de hidroginástica em pelo menos parte dos desfechos avaliados. O resultado da metanálise apontou para uma melhora significativa nos testes funcionais flexão de cotovelo, levantar e sentar, alcançar atrás das costas, sentado e alcançar e foot up and go. A melhora destes testes reflete um incremento da força muscular resistente de membros 
a

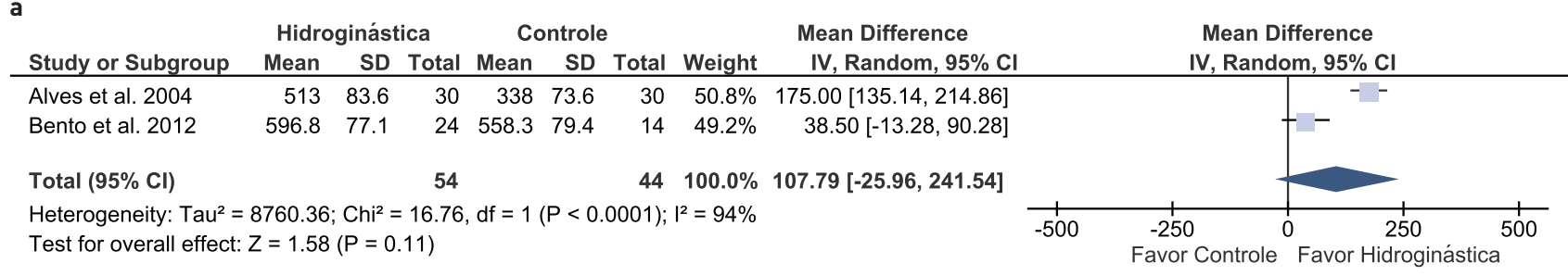

b

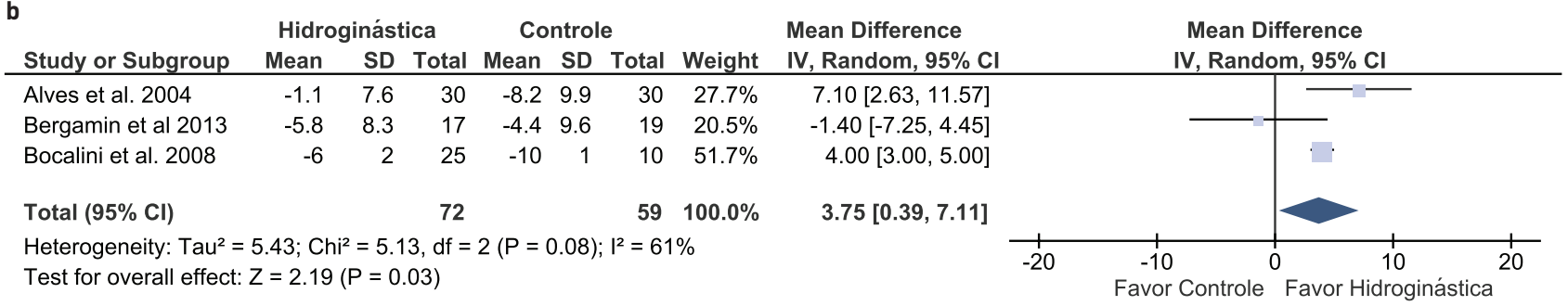

FIGURA 4 - Efeito da hidroginástica no teste alcançar atrás das costas (a) e sentado e alcançar (b).

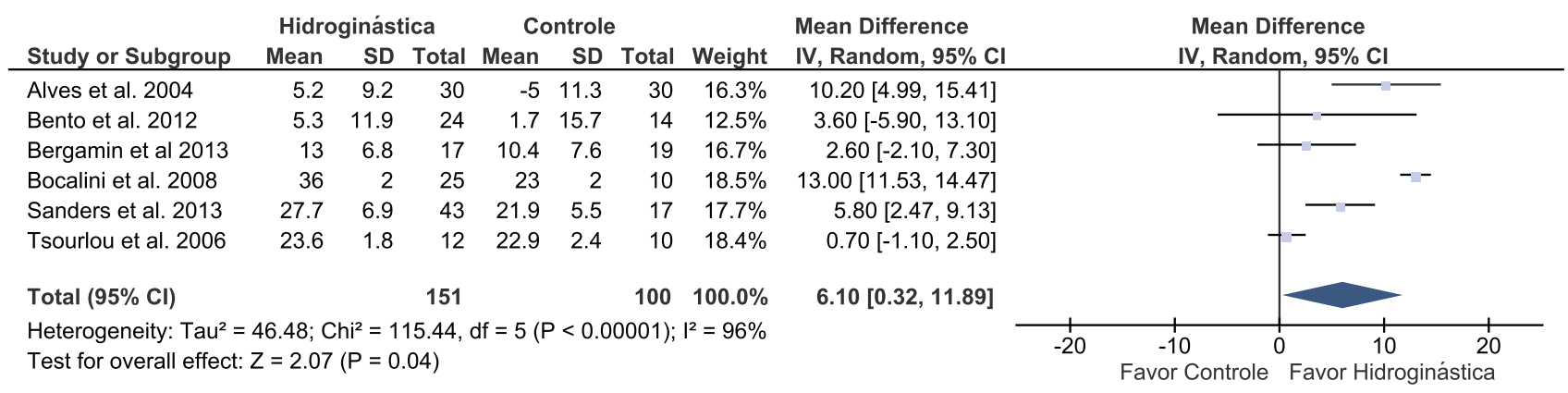

FIGURA 5 - Efeito da hidroginástica no teste foot up and go.

superiores e inferiores, da flexibilidade de membros superiores e inferiores e da agilidade e equilíbrio dinâmico, respectivamente, de indivíduos idosos.

A hidroginástica vem sendo fortemente recomendada para a população idosa por proporcionar uma atividade física em um ambiente mais seguro quando comparado ao meio terrestre. Desta forma, o praticante se beneficia com os efeitos benévolos do exercício físico enquanto apresenta um menor stress articular $^{8}$ e cardiovascular ${ }^{9}$, além de um menor risco de sofrer alguma lesão. Essas características são de grande importância para os idosos, visto que é indicado que este público realize exercício físico afim de amenizar os efeitos deletérios do envelhecimento; contudo, frequentemente apresentam desordens osteoarticulares ou cardíacas que limitam a sua capacidade de se exercitar em meio terrestre.

Dentre os efeitos deletérios do envelhecimento, a perda de massa e de força muscular recebem grande destaque devido às suas sérias implicações, sendo considerados os principais fatores que levam à perda da mobilidade funcional ${ }^{20}$ e de independência do idoso ${ }^{7}$.

O resultado da presente metanálise demonstrou que a prática de hidroginástica, em uma frequência semanal de duas a três sessões, promove um incremento significativo da força muscular resistente de membros superiores e inferiores em idosos. Os estudos de Alves et al. ${ }^{10}$, Bento et al. ${ }^{11}$ e Sanders et al. ${ }^{16}$ possuíam uma prescrição de treinamento específica para o desenvolvimento da força muscular, logo, o aumento desta variável já era esperado. 
Contudo, os estudos de Bocalini et al. ${ }^{13}$ e Rica et al. ${ }^{15}$ apresentavam apenas uma prescrição de treinamento aeróbia, sugerindo que o treinamento aeróbio de hidroginástica pode promover ganhos de força resistente. $\mathrm{O}$ incremento dessa variável após um treinamento no meio aquático pode ser explicado pela repetição de um exercício por um longo período de tempo em altas velocidades. Isso maximiza a resistência ao avanço $\left(R=0,5 \rho \mathrm{A} \mathrm{v}^{2} \mathrm{Cd}\right.$, em que, $\rho$ é a densidade do fluído, A é a área projetada, v é a velocidade do movimento e $\mathrm{Cd}$ é o coeficiente de arrasto) ${ }^{21}$, podendo gerar estímulos de força resistente ${ }^{22}$. No entanto, deve-se atentar que esse resultado foi encontrado em idosos previamente sedentários, podendo não ser reprodutível em indivíduos já treinados.

$\mathrm{O}$ achado do presente estudo corrobora o resultado da revisão sistemática de Bergamin et al. ${ }^{12}$, fortalecendo a evidência de que a prática de hidroginástica promove ganhos de força muscular em idosos.

$\mathrm{O}$ avanço da idade também é marcado por uma diminuição da capacidade aeróbia. Estima-se que ocorra uma redução da potência aeróbia em $10 \%$ por década a partir dos $20 \operatorname{anos}^{3}$. A fim de amenizar este declínio, o exercício físico aeróbio tem sido recomendado. Contrariando a literatura, a presente metanálise revelou que o treinamento de hidroginástica não promoveu incrementos na capacidade aeróbia, avaliada pela distância percorrida no teste de seis minutos de caminhada. Apesar disto, demonstrou-se que a hidroginástica proporcionou uma manutenção importante desta variável. No entanto, este achado deve ser analisado com cautela, pois somente dois estudos foram incluídos na metanálise e observou-se uma alta heterogeneidade $\left(\mathrm{I}^{2}=94 \%\right)$.

Discordando do achado do presente estudo, na revisão sistemática de Bergamin et al. ${ }^{12}$ concluiu-se que o treinamento de hidroginástica promove incrementos na capacidade aeróbia. A discordância entre os resultados pode ser explicada pelas diferentes formas de predizer a capacidade cardiorrespiratória. Enquanto o presente estudo avaliou o desempenho em um teste de caminhada de seis minutos, o estudo citado avaliou esse desfecho pelo consumo de oxigênio de forma direta.

O presente estudo também demonstrou que a prática de hidroginástica proporciona uma melhora da flexibilidade de membros superiores e inferiores de idosos previamente sedentários. Nenhum dos estudos incluídos na análise deste desfecho incluiu exercícios específicos para o desenvolvimento desta variável em seu treinamento. Contudo, em todos eles foram realizados exercícios de alongamento como aquecimento ou volta à calma e, além disso, os exercícios da parte principal da aula foram realizados em grandes amplitudes de movimento. Sendo assim, esses dois fatores podem explicar os ganhos neste desfecho após o período de treinamento. O presente achado corrobora o resultado da revisão de Bergamin et al. ${ }^{12}$, que também abrangeu estudos que avaliaram a flexibilidade por meio de testes funcionais. Este é um achado importante na medida em que indivíduos que apresentam níveis adequados de flexibilidade possuem maior mobilidade articular, melhorando o seu desempenho nas AVDs e tornando-os mais independentes.

Por fim, observou-se um efeito benéfico do treinamento de hidroginástica sobre o tempo necessário para completar o teste foot up and go e timed up and go. Esses testes avaliam o equilíbrio dinâmico e a agilidade do indivíduo. Desta forma, conclui-se que a prática de hidroginástica promove a melhora do equilíbrio dinâmico e da agilidade em idosos previamente sedentários. De 
fato, estudos têm sugerido que o meio aquático, por criar um ambiente de instabilidade pode promover ganhos no equilíbrio corporal ${ }^{23,24}$. Este parece ser um dado inovador na literatura, visto que não foi encontrada outra revisão sistemática sobre os efeitos da hidroginástica sobre o equilíbrio corporal.

A melhora do equilíbrio pode representar um menor risco de quedas ${ }^{23}$, o que é de extrema importância para esta faixa etária, visto que as quedas são fenômenos frequentes: cerca de $30 \%$ da população com mais de 65 anos cai anualmente ${ }^{25}$.Ademais, as quedas possuem consequências graves: podem levar à incapacidade, mantendo o idoso acamado, e até à morte, sendo responsáveis por $70 \%$ das mortes acidentais em pessoas com mais de 75 anos $^{26}$.

Os resultados do presente estudo demonstram que a prática de hidroginástica promove uma melhora da capacidade funcional, por meio do incremento de força resistente, flexibilidade, equilíbrio dinâmico e agilidade. Desta forma esta modalidade pode ser indicada como uma alternativa para aqueles indivíduos que necessitam melhorar a sua capacidade funcional. No entanto, sugere-se a realização de mais estudos nesta área, devido à alta heterogeneidade existente entre os estudos aqui abordados.

\section{CONCLUSÃO}

A prática de hidroginástica pode ser recomendada com a finalidade de melhorar a capacidade funcional de idosos, uma vez que esta modalidade promove o incremento da força resistente e da flexibilidade de membros superiores e inferiores e do equilíbrio dinâmico. Este resultado representa uma melhora na capacidade de realizar as AVDs, promovendo uma maior independência funcional ao idoso.

\section{REFERÊNCIAS}

1. Instituto Brasileiro de Geografia e Estatística - IBGE. Censo 2010. [Citado em 2012 out 23]. Disponível em: www.censo2010.ibge.gov.br

2. Organização Mundial de Saúde - OMS. [Citado em 2012 out 22]. Disponível em: <http://www.who.int/countries/bra/es/>.

3. Wilmore JH, Costill DL, Kenney WL. Physiology of Sports and Exercise. United States: Human Kinetics, 2010.

4. Tsourlou T, Benik A, Dipla K, Zafeiridis A, Kellis S. The effects of a twenty-four-week aquatic training program on muscular strength performance in healthy elderly women. J Strength Cond Res. 2006; 20(4): 811-18.

5. Kaneda K, Wakabayashi H, Sato D, Nomura T. Lower extremity muscle activity during different types and speeds of underwater movement. J Physiol Anthropol. 2008; 26(2):197-200.

6. Holland GJTK, Shigematsu R, Nakagaichi M. Flexibility and physical functions of older adults: a review. J Aging Phys Activ. 2002; 10(2):169-206.

7. Power SK, Howley ET. Fisiologia do Exercício: teoria e aplicação ao condicionamento e ao desempenho. São Paulo: Manole, 2000.

8. Alberton CL, Finatto P, Pinto SS, Antunes AH, Cadore EL, Tartaruga M, et al. Vertical ground reaction force responses to different head-out aquatic exercises performed in water and on dry land. J Sports Sci. 2014; 30:1-11.

9. Alberton CL, Tartaruga MP, Pinto SS, Cadore EL, Silva EM, Kruel LFM. Cardiorespiratory responses to stationary running at different cadences in water and on land. J Sports Med Phys Fit. 2009; 49: 42-51.

10. Alves RV, Mota J, Costa MC, Alves JGB. Aptidão física relacionada à saúde de idosos: influência da hidroginástica. Rev Bras Med Esp. 2004; 10(1): 31-7. 
11. Bento PC, Pereira G, Ugrinowitsch C, Rodacki AL. The effects of a water-based exercise program on strength and functionality of older adults. J Aging Phys Act. 2012; 20(4):469-83.

12. Bergamin M, Ermolao A, Tolomio S, Berton L, Sergi G, Zaccaria M. Water- versus land-based exercise in elderly subjects: effects on physical performance and body composition. Clin Interv Aging. 2013; 8:1109-17.

13. Bocalini DS, Santos L, Serra AJ. Physical exercise improves the functional capacity and quality of life in patients with heart failure. Clinics. 2008; 63:437-42.

14. Katsura Y, Yoshikawa T, Ueda SY, Usui T, Sotobayashi D, Nakao H, et al. Effects of aquatic exercise training using water-resistance equipment in elderly. Eur J Appl Physiol. 2010; 108(5):957-64.

15. Rica RL, Carneiro RM, Serra AJ, Rodriguez D, Pontes Junior FL, Bocalini DS. Effects of water-based exercise in obese older women: impact of short-term follow-up study on anthropometric, functional fitness and quality of life parameters. Geriatr Gerontol Int. 2013; 13(1):209-14.

16. Sanders ME, Takeshima N, Rogers ME, Colado JC, Borreani S. Impact of the S.W.E.A.T. ${ }^{\text {TM }}$ water-exercise method on activities of daily living for older women. J Sports Sci Med. 2013; 12(4): 707-15.

17. Bergamin M, Zanuso S, Alvar B, Ermolao A, Zaccaria M. Is water-based exercise training sufficient to improve physical fitness in the elderly? Eur Rev Aging Phys Activ. 2012; 9:129-41.

18. Higgins J, Green S. Cochrane handbook for systematic reviews of interventions 5.0. Chichester, 2011.

19. Moher D, Liberati A, Tetzlaff J, Altman DG, Group P. Preferred reporting items for systematic reviews and meta-analyses: the PRISMA statement. J Clin Epidemiol. 2009; 62(10):1006-12.

20. DoHerty TJ. Invited review: Aging and sarcopenia. J Appl Physiol. 2003; 95:1717-27.

21. Alexander R. Mechanics and energetics of animal locomotion. In: Swimming, R. Alexander and G. Goldspink. London: Chapman and Hall. 222-248, 1977.

22. Kanitz AC, Delevatti RS, Reichert T, Liedtke GV, Ferrari R, Almada BP, et al. Effects of two deep water training programs on cardiorespiratory and muscular strength responses in older adults. Exp Gerontol. 2015; 64:55-61.

23. Melzer I, Elbar O, Tsedek I, Oddsson LI. A water-based training program that include perturbation exercises to improve stepping responses in older adults: study protocol for a randomized controlled cross-over trial. BMC Geriatrics. 2008.

24. Avelar NCP, Bastone AC, Alcântara MA, Gomes WF. Efetividade do treinamento de resistência à fadiga dos músculos dos membros inferiores dentro e fora d'água no equilíbrio estático e dinâmico de idosos. Rev Bras Fisioter. 2010; 14(3): 229-36.

25. Perracini MR, Ramos LR. Fatores associados a quedas em uma coorte de idosos residentes no comunidade. Rev Saúde Pública. 2002; 36(6): 709-16.

26. Bittar RSM, Pedalini MEB, Bottino MA \& Formigoni LG. Síndrome do desequilíbrio no idoso. Pró Fono. 2002; 14(1): 119-28.

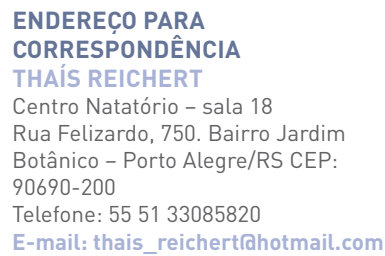

